\title{
Preliminary study of a microsporidian infecting the teleost fish Cephalopholis hemistiktos from the Red Sea in Saudi Arabia
}

\author{
Azevedo, C. ${ }^{* * *}$, Rocha, S., Abdel-Baki, A.A. ${ }^{* * *}$, Al-Quraishy, S. ${ }^{* * *}$ and Casal, G., ${ }^{* * * * *}$ \\ * Interdisciplinary Centre of Marine and Environmental Research (CIIMAR/UP), University of Porto, \\ PORTUGAL. \\ ** Institute of Biomedical Sciences Abel Salazar (ICBAS/UP), University of Porto, PORTUGAL. \\ *** Zoology Department, College of Sciences, King Saud University, Riyadh, SAUDI ARABIA \\ **** Department of Sciences, High Institute of Health Sciences - North (CESPU), Gandra, PORTUGAL. \\ Email: azevedoc@icbas.up.pt
}

Microsporidia are obligatory intracellular parasites of medical and commercial importance, characterized by a severe reduction, or even absence, of the cellular components typical of eukaryotes. Presently, there are about 156 microsporidian species grouped within 18 genera that infect marine and freshwater fishes worldwide [1]. The present study uses combined ultrastructural and molecular data to describe the first species of Glugea reported from the Red Sea, Jeddah, Saudi Arabia.

A parasitological study of the marine teleost fish, Cephalopholis hemistiktos Rüppell, 1830 (Serranidae: Epinephelinae), collected from the Red Sea, revealed several xenomas localized in the intestinal wall of several specimens. Spores were measured using the Nomarski differential interference contrast optic (DIC) microscopy, and prepared for transmission electron microscopy, as well as for molecular procedures. Extraction of genomic DNA was performed using the GenEluteTM mammalian genomic DNA kit (Sigma) and the region of the SSU, ITS and LSU (partial) rRNA gene amplified using two pairs of specific primers: V1F/HG5F_rev and HG4F/HG4R. After sequencing, the fragments from both strands were aligned using Clustal W in MEGA 5 software. Distance estimation was performed for the Kimura 2-parameter model and the Maximum Likelihood phylogenetic tree constructed.

Whitish spherical cysts, about $2 \mathrm{~mm}$ in diameter, appeared adjacent to the epithelial cells of the intestine. Cysts encapsulated by a thick and host-derived fibrous membrane. Synchronous, the cytoplasm contained many monomorphic mature spores within parasitophorous vacuoles (Fig. 1). Mature spores ovoid to pyriform, about 5.0 (4.0-6.0) $\mu \mathrm{m}$ long and 2.2 (2.0-3.0) $\mu \mathrm{m}$ wide (Fig. 2). Polar filament isofilar, surrounded by a lamellar polaroplast at the spore's anterior pole, and forming 26-29 coils organized in three rows at the spore's posterior pole (Fig. 3). Nucleus positioned between polaroplast and posterior vacuole. Posterior vacuole surrounded by the polar filament coils, irregular and containing masses of electron-dense material. The posterosome was never observed.

The ultrastructural organization of the xenomas and spore, combined with the molecular data, suggest that this parasite belongs to the family Glugeidae, more specifically to the genus Glugea Thélohan, 1891. Comparison to other Glugea spp. further suggests this parasite as a new species (Fig. 4).

This work was partially supported by FCT (Lisbon, Portugal) within the scope of the project DIRDAMyx, reference PTDC/MAR/116838/2010; Eng. ${ }^{\circ}$ António Almeida Foundation (Porto, Portugal); and the project no. RGP-VPP-002 of King Saud University (Riyadh, Saudi Arabia).

[1]Lom, J. Systematic Parasitology, 53, 81-99, 2002. 


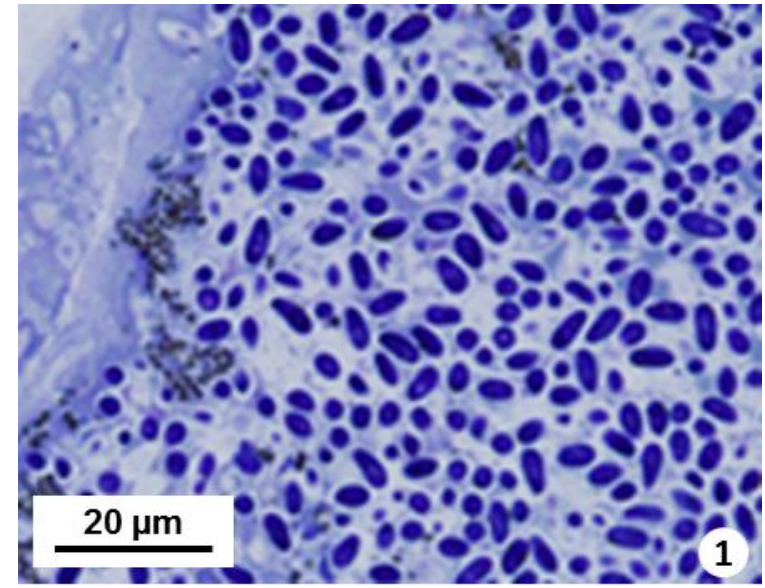

Fig. 1 - Semithin section of a xenoma showing numerous mature spores contained within parasitophorous vacuoles.

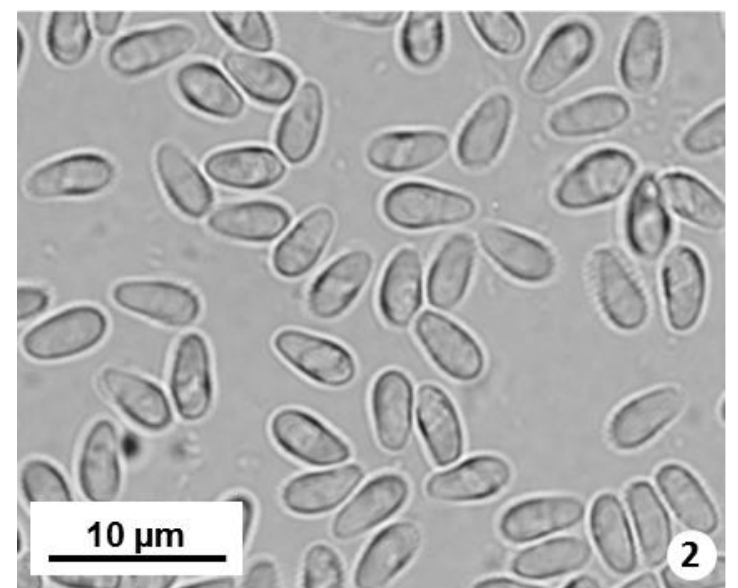

Fig. 2 - Free fresh mature spores observed in DIC optics.
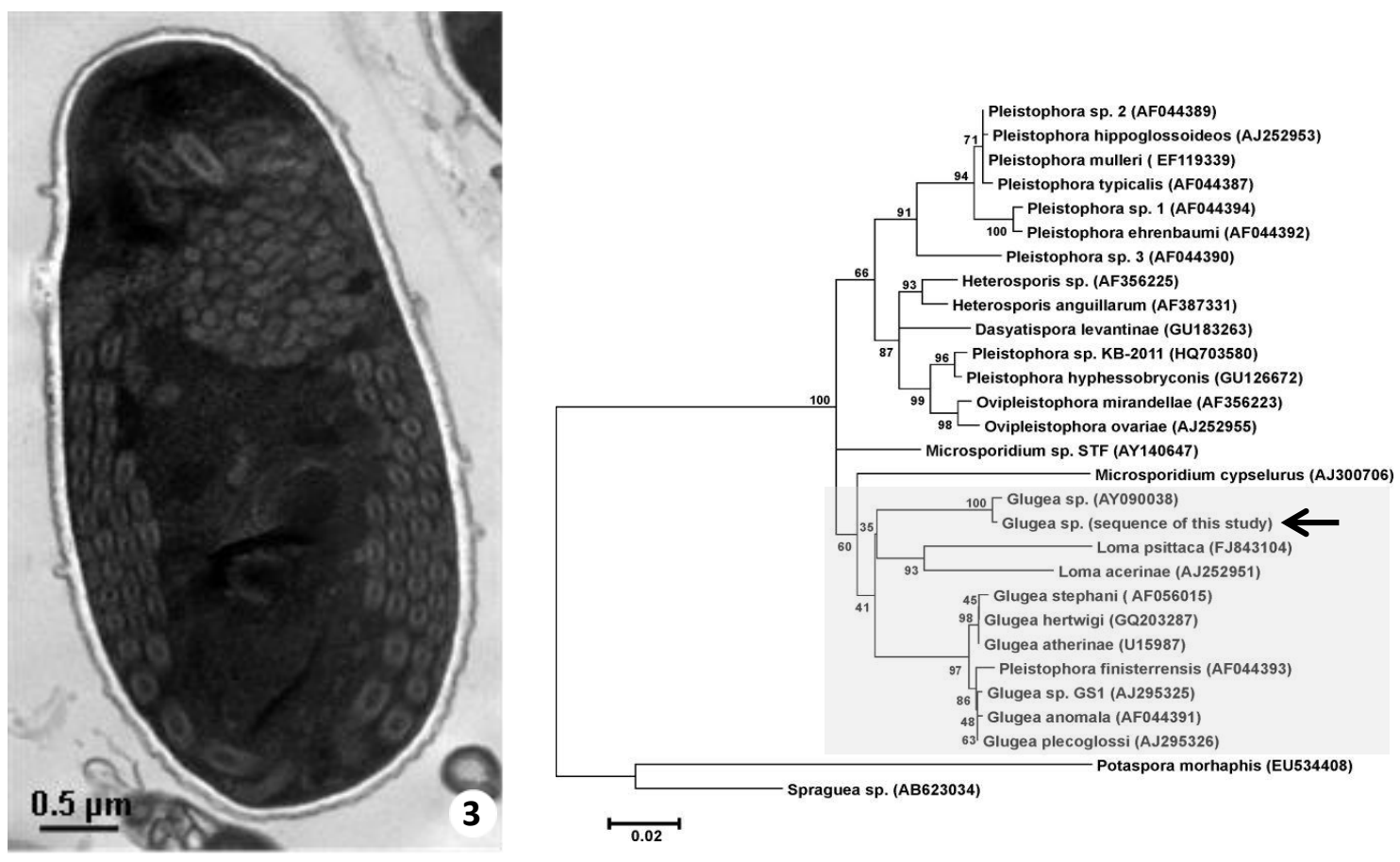

Fig. 3 - Longitudinal section of a spore showing the polar filament forming 26-29 coils organized in three rows at the spore's posterior pole.
Fig. 4 - Maximum Likelihood tree for the SSU, ITS and partial LSU rDNA sequences of Glugea sp. and other selected microsporidians. GenBank accession numbers in parentheses after the species name; scale given under the tree. 